\title{
Delivering Cognitive Stimulation Therapy (CST) Virtually: Developing and Field-Testing a New Framework
}

\author{
Luke Perkins', Emily Fisher (D)', Cerne Felstead', Claire Rooney ${ }^{2}$, Gloria HY Wong $\mathbb{D}^{3}$, Ruizhi Dai (iD ${ }^{4}$, \\ Sridhar Vaitheswaran $\mathbb{D}^{5}$, Nirupama Natarajan $\mathbb{D}^{5}$, Daniel C Mograbi $\mathbb{D}^{6,7}$, Cleusa P Ferri ${ }^{8,9}$, \\ Joshua Stott $\mathbb{D}^{\prime}$, Aimee Spector' \\ 'Research Department of Clinical, Educational and Health Psychology, University College London, London, UK; ' ${ }^{2}$ Occupational Therapy Department, \\ Older Persons Services, Our Lady's Hospice and Care Services, Harold's Cross, Dublin, Ireland; ${ }^{3}$ Department of Social Work and Social \\ Administration, University of Hong Kong, Hong Kong, Hong Kong; ${ }^{4}$ Faculty of Social Sciences, University of Hong Kong, Hong Kong, Hong Kong; \\ ${ }^{5}$ Dementia Care in Schizophrenia Research Foundation (DEMCARES), Chennai, Tamil Nadu, India; ${ }^{6}$ Department of Psychology, Pontifícia Universidade \\ Católica do Rio de Janeiro, Rio de Janeiro, Brazil; 'Institute of Psychiatry, Psychology and Neuroscience, King's College London, London, UK; \\ ${ }^{8}$ Department of Psychiatry, Universidade Federal de São Paulo, São Paulo, Brazil; ' ${ }^{9}$ Health Technology Assessment Unit, Hospital Alemão Oswaldo \\ Cruz, São Paulo, Brazil
}

Correspondence: Emily Fisher, Research Department of Clinical, Educational and Health Psychology, University College London, Gower Street, London, WCIE 6BT, UK, Tel +44 207679 5770, Email emily.fisher@ucl.ac.uk

Purpose: This feasibility and pilot study aimed to develop and field-test a 14-session virtual Cognitive Stimulation Therapy (vCST) programme for people living with dementia, developed as a result of services moving online during the COVID-19 pandemic.

Methods: The vCST protocol was developed using the existing group CST manual, through stakeholder consultation with people living with dementia, caregivers, CST group facilitators and dementia service managers. This protocol was then field-tested with 10 groups of people living with dementia in the Brazil, China (Hong Kong), India, Ireland and the UK, and feedback on the protocol was gathered from 14 facilitators.

Results: Field testing in five countries indicated acceptability to group facilitators and participants. Feedback from these groups was used to refine the developed protocol. The final vCST protocol is proposed, including session materials for delivery of CST over videoconferencing and a framework for offering CST virtually in global settings.

Conclusion: vCST is a feasible online intervention for many people living with dementia. We recommend that it is offered to those unable to access traditional in-person CST for health reasons, lack of transport or COVID-19 restrictions. Further research is needed to explore if participant outcomes are comparable to in-person CST groups.

Keywords: dementia, COVID-19, psychosocial intervention, telehealth

\section{Introduction}

There are 55 million people living with dementia across the globe so there is a significant need to develop interventions that tackle the physical, mental, social and financial impacts of the condition. ${ }^{1}$ With no treatments that prevent the progressive neurological deterioration resulting from dementia, the need for supportive interventions is pressing.

Cognitive Stimulation Therapy (CST) is the best-established psychological intervention for people living with dementia and has demonstrated benefits in relation to cognition, quality of life and overall cost-effectiveness. ${ }^{2}$ CST was developed by Spector et al. ${ }^{3}$ as a brief, 14-session face-to-face intervention for people living with dementia, comprised of group activities which stimulate cognition including memory and language. In the United Kingdom (UK), CST is recommended by the National Institute for Health and Social Care Excellence (NICE) as a key 
psychosocial intervention for dementia. ${ }^{4}$ Culturally adapted versions of CST have been successfully implemented across the globe and CST is recommended internationally for people with early-stage dementia. ${ }^{5-7}$

Access to in-person interventions like CST was immediately and unexpectedly restricted when the COVID-19 pandemic spread throughout the world in early $2020 .{ }^{8}$ Not only did this prevent access to services, treatments and interventions for people living with dementia who had been attending them previously, it also highlighted the gap in service provision for those who could not access services outside of the pandemic context. ${ }^{9}$ This includes people living with dementia residing in rural communities who are unable to readily access transport or those with reduced mobility. In response to the pandemic, many services have had to adapt rapidly in order to keep services accessible whilst maintaining the need for social distancing. This has seen services turn to developing treatments and interventions that can be delivered using digital technology. ${ }^{9}$

\section{Digital Technology in Psychological Interventions}

The use of digital technology or "e-Health" has become popular in clinical populations, such as adults with anxiety and depression, even before its mass adoption during the pandemic. ${ }^{10}$ E-Health is defined by the World Health Organization as 'the cost-effective and secure use of information and communications technologies in support of health and healthrelated fields'. ${ }^{11}$ Leading up to the pandemic, most of the digital psychological interventions on offer were based on Cognitive Behavioural Therapy (CBT), although other psychological therapies have been demonstrated to be beneficial when delivered virtually. ${ }^{12-14}$

There is increasing interest in the use of e-health interventions for people living with dementia, and evidence of feasibility and their impact on psychological, social and cognitive domains. ${ }^{15-17}$ Existing research does highlight the challenges that older adults face in accessing e-health interventions. Older adults can find access more difficult than working age adults due to barriers including limited computer literacy and lack of trust in digital interventions. ${ }^{18,19}$ This is further compounded in people living with dementia who face declining cognitive ability and independent day-to-day functioning, and reduced help-seeking due to beliefs that cognitive symptoms are a normal part of aging. ${ }^{20,21}$ Despite these barriers, it appears to be both feasible and beneficial to continue to deliver psychological interventions during the pandemic and beyond..$^{22,23}$

These findings pave the way for further expansion of e-health application to a wide variety of existing programmes for people living with dementia. CST is one such intervention that could benefit from adaptation for online use, especially in the context of the COVID-19 pandemic where in-person services may be restricted. The existing literature related to virtual delivery of CST is limited to a single case study series. ${ }^{24}$ Furthermore, a workshop hosted by the [redacted for review] identified the demand from CST practitioners for a standard protocol for delivery of virtual CST, to enable service continuation during the pandemic. Therefore, the main aims of this study are:

1. To develop a protocol for virtual CST (vCST).

2. To evaluate the feasibility and acceptability of the vCST protocol through field-testing in a range of settings and countries.

3. To provide a framework for offering vCST virtually across global settings.

\section{Materials and Methods}

\section{Overview}

The study followed the Medical Research Council's 'developing and evaluating complex interventions' framework, made up of four phases: Development, Feasibility, Evaluation, and Implementation. ${ }^{25}$ Here we focus only on the Development and Feasibility phases. The project was split into two parts: (a) Development of the vCST intervention using the existing CST group manual and stakeholder consultation; ${ }^{26}$ and (b) Feedback and validation from CST facilitators following vCST protocol field-testing in Brazil (Rio de Janeiro and São Paulo), China (Hong Kong), India (Chennai), Ireland and the UK. 


\section{Ethics}

This study was conducted in accordance with the Declaration of Helsinki. Ethical approval was granted in all countries (UK and Ireland, UCL Research Ethics Committee, project 17127/001; India, Schizophrenia Research Foundation [SCARF], SRF-DC/18/OCT-2020; Brazil, Federal University of São Paulo [UNIFESP] and CONEPE, process no. 4895729 and Federal University of Rio de Janeiro Institute of Psychiatry, ID 57019616.5.1001.5263; Hong Kong, University of Hong Kong, reference no. EA2004006).

\section{Part I: Development of vCST Protocol}

The vCST protocol was developed by adapting the existing group CST manual, informed by stakeholder consultations with four groups: people living with dementia; caregivers of people living with dementia; service managers; and CST group facilitators who had previously run vCST groups. ${ }^{26}$ People with dementia and caregivers were service users of organisations in the UK, and service managers were recruited from these same services. Facilitators were recruited by email from third-sector organisations in the UK and Hong Kong.

Questions for the consultations were developed using the Consolidated Framework for Implementation Research (CFIR). ${ }^{27}$ Interview questions related to the five main domains of the framework: intervention characteristics, outer setting, inner setting, characteristics of individuals and process.

All focus groups took place over the online video conferencing platform "Zoom" and were recorded for post-group analysis. Two researchers then took field notes from these recordings, which were clustered together into key ideas. The data gathered from the focus groups was used to develop guidelines for delivering vCST in collaboration with researchers at The University of Hong Kong working on a trial of vCST. Both teams worked closely throughout the project to ensure the vCST intervention and protocols were aligned, whilst also accounting for specific cultural adaptations.

\section{Part 2: Field-Testing \\ Design}

The vCST protocol was field tested in five countries and feedback was gathered from facilitators. vCST was delivered through a randomised controlled trial in the UK and Ireland. In Brazil, Hong Kong, and India, vCST was delivered as a pre-post study. The empirical results from these studies will be reported separately. Here we focus on the content of the vCST protocol and its feasibility through field-testing.

\section{Procedure}

The template version was co-developed in the UK, Ireland and Hong Kong as described above, then translated and culturally adapted by teams in India and Brazil, based on previously culturally adapted versions of in-person CST. ${ }^{5,28}$ In India, additional stakeholder engagement was carried out to ensure the intervention was acceptable and culturally appropriate. Interviews with psychologists, psychiatrists and healthcare professionals took place prior to vCST groups beginning. Interviews with people living with dementia and caregivers took place prior to and midway through the delivery of the intervention, where delivery could be adjusted according to feedback.

Facilitators all had experience of working directly with people living with dementia and had experience of either delivering or observing in-person CST. Facilitators were asked for feedback following completion of the vCST sessions, which comprised:

1. Which sessions/activities went well?

2. Which sessions/activities did not go as well?

3. Did any activities need adapting further?

4. Did you develop any new ideas?

5. Any other comments. 


\section{Results}

\section{Part I: Development of vCST Protocol}

\section{Stakeholder Consultation}

Twenty participants across four focus groups took part in the consultation. Each focus group lasted approximately 90 minutes and was facilitated by two researchers. Group one was attended by three people living with dementia from the same third-sector organisation in the south-west of England. An additional individual session was conducted by the researcher with a fourth person with dementia from a different third-sector organisation in the north of England. All four had attended in-person group CST sessions before the first UK national COVID-19 lockdown, before switching to virtual CST groups midway through the intervention.

Group two was attended by four caregivers of people living with dementia. Three were recruited from a third-sector organisation in the south-east of England and one from a third sector organisation in the south-west of England. Only one caregiver had cared for a person living with dementia who had previously attended vCST before.

Group three was attended by eight vCST facilitators. One was from Hong Kong team and all others were from England. All were working in third-sector dementia organisations and had facilitated vCST sessions online previously.

Group four was attended by four service managers recruited from third-sector dementia organisations across England - one of whom worked for a service that had already implemented vCST sessions. The key ideas relating to each of the CFIR domains are outlined in Table 1.

The vCST protocol developed through stakeholder consultation follows the 14-session plan outlined in the group CST manual in terms of session structure and themes, with activities adapted for online delivery. ${ }^{26}$ Sessions last $45-60$ minutes as stated in the manual. The final order of sessions matched the group CST manual as outlined in Table 2.

All vCST sessions begin with introductions, welcoming members to the group, orienting participants to time, date and place and, doing a warm-up activity. Everyone then sings the chosen group song and discusses a recent newspaper article. Next, group members choose a main activity based on the session theme. Each session ends with a summary, feedback from participants, reminding participants of the next session theme, including activities and materials to bring, and then saying goodbye.

\section{Session Format}

Each group should run with four to five participants. This was deemed as the optimum group size given the number of people who would be visible on the screen at one time, and to enable the participation of all group members. Sessions should run for 45-60 minutes dependent on the group's engagement levels during the session. Participants should be able to take breaks during session if they feel unable to stay engaged but should be encouraged to stay for the whole session if possible.

\section{Session Activities}

In-person CST uses physical objects, pictures, and music. For vCST, activities should be taken from the group CST manual and be transferred into a digital format. Text, images, videos, and sounds can be presented to all participants through software such as Microsoft PowerPoint, by using the share screen and/or share sound functions. For example, in the "Using Money" session interactive PowerPoint presentations of a price matching task can be used, and for the "Current Affairs" session, online news clips can be shown to the group by sharing the screen and sound.

\section{Physical Resources}

The initial protocol was based on the assumption that researchers were unable to send physical resources for sessions to participants. Any additional resources required, such as pens, paper, or other household objects, should be agreed with participants at the end of the previous session. This would give participants time to gather these objects if needed. Each session contained at least one activity that did not need additional resources, in case participants were not able to obtain them. 
Table I Key Questions and Ideas from Focus Groups

\begin{tabular}{|c|c|c|}
\hline Stakeholders & Key Questions & Key Ideas \\
\hline \multicolumn{3}{|r|}{ CFIR domain: "Intervention” } \\
\hline PLWD & $\begin{array}{l}\text { I. What aspects of CST need to be different to } \\
\text { implement it online? }\end{array}$ & $\begin{array}{l}\text { I. Sufficient access to technology needed. Access to physical objects for multisensory component of activities is limited but } \\
\text { sessions still work well without this. Smaller group size needed - optimal number of participants is four. }\end{array}$ \\
\hline Caregivers & $\begin{array}{l}\text { I. What aspects of CST need to be different to } \\
\text { implement it online? }\end{array}$ & $\begin{array}{l}\text { I. Access to physical objects is limited. Group members could bring objects themselves or facilitators could post them out. } \\
\text { PLWD may need support using computer technology. One-to-one training sessions could be offered before groups begin. } \\
\text { Facilitators should be more directive and should simplify discussions. Caregivers should also provide support. }\end{array}$ \\
\hline Facilitators & $\begin{array}{l}\text { I. What is the optimal group size? } \\
\text { 2. Which sessions work well virtually and which } \\
\text { need adapting? } \\
\text { 3. Are session handouts helpful in vCST? } \\
\text { 4. Can services offer the recommended CST } \\
\text { dose? (Twice weekly for } 7 \text { weeks) } \\
\text { 5. Which video conferencing app is best? }\end{array}$ & $\begin{array}{l}\text { I. Optimum group size is four to five. Smaller groups may support engagement. } \\
\text { 2. Sessions that are mainly discussion-based work well as they require no extra resources or adaptations. Sessions with } \\
\text { multisensory components are harder to deliver without physical objects. Adaptations for online delivery include: Show images } \\
\text { using "share screen"; Group members bring objects to supplement discussions; Listen to a song together. } \\
\text { 3. Handouts could supplement sessions, but participants may be unable to print and/or facilitators unable to post them. } \\
\text { 4. Resource restrictions in services may make offering the recommended dose difficult, but online delivery makes it easier as } \\
\text { participants do not need to travel. } \\
\text { 5. Zoom is the most popular and widely used app. It has interactive features ("share screen", "whiteboard" and "clap" } \\
\text { functions). }\end{array}$ \\
\hline Service Managers & $\begin{array}{l}\text { I. What are the advantages and disadvantages of } \\
\text { implementing vCST in services? } \\
\text { 2. What investments are needed for services to } \\
\text { implement vCST? }\end{array}$ & $\begin{array}{l}\text { I. Advantages: Possible increased attendance due to removal of travel and preparation time. vCST is relatively cheap to run. } \\
\text { Disadvantages: Less peer support for caregivers who will not spend time together whilst PLWD attend sessions. Caregivers } \\
\text { may need to provide technology support and could become too involved in the sessions. Participants' lack of experience with } \\
\text { technology could negatively affect recruitment or engagement in sessions. Facilitating group cohesion may be harder - } \\
\text { facilitators should be directive, encourage group participation and a second facilitator should support. } \\
\text { 2. Training staff in vCST delivery, allocating time for staff to prepare and evaluate sessions, obtaining suitable technology for } \\
\text { group members and facilitators. PLWD must have access to a laptop or tablet to make sure the screen is large enough. }\end{array}$ \\
\hline \multicolumn{3}{|r|}{ CFIR domain: “Outer Setting” } \\
\hline PLWD & $\begin{array}{l}\text { I. Are interventions available for PLWD unable to } \\
\text { attend services in person? } \\
\text { 2. Would you participate in vCST? What would } \\
\text { influence this decision? }\end{array}$ & $\begin{array}{l}\text { I. PLWD not aware of online interventions or services, but felt they were necessary to increase access. } \\
\text { 2. Open to attending vCST if sufficient information given beforehand such as testimonials from previous attendees, being } \\
\text { shown clips or demonstrations of previous sessions, or attending with someone they knew. }\end{array}$ \\
\hline Caregivers & $\begin{array}{l}\text { I. Are interventions available for PLWD unable to } \\
\text { attend services in person? } \\
\text { 2. Would PLWD participate in vCST? What would } \\
\text { influence this decision? }\end{array}$ & $\begin{array}{l}\text { I. There are currently online groups for caregivers, or for caregivers and PLWD. Not aware of online groups solely for PLWD. } \\
\text { 2. Offer of vCST likely to be taken up by PLWD due to perceived benefits of brain stimulation; however, this would need to } \\
\text { be initiated and organised by caregivers. }\end{array}$ \\
\hline
\end{tabular}


Table I (Continued).

\begin{tabular}{|c|c|c|}
\hline Stakeholders & Key Questions & Key Ideas \\
\hline \multicolumn{3}{|r|}{ CFIR domain: “outer setting” (continued) } \\
\hline Facilitators & $\begin{array}{l}\text { I. To what extent can PLWD independently } \\
\text { access vCST? }\end{array}$ & $\begin{array}{l}\text { I. PLWD may independently use video conferencing with experience, but most need caregiver support to set it up. Caregivers } \\
\text { are essential in ensuring PLWD can access and participate in vCST. However, they should not participate themselves or } \\
\text { contribute on behalf of the people they care for. }\end{array}$ \\
\hline Facilitators & $\begin{array}{l}\text { 2. What factors/characteristics of PLWD affect } \\
\text { their ability to engage in vCST? }\end{array}$ & $\begin{array}{l}\text { 2. Factors include aphasia, visual or auditory impairments, more severe levels of dementia, and low levels of digital literacy. } \\
\text { The use of headphones or using a bigger screen may be necessary. Videos or testimonials from previous sessions may } \\
\text { encourage new members to attend if they were not confident or had concerns about attending. }\end{array}$ \\
\hline Service Managers & $\begin{array}{l}\text { I. Are interventions available for PLWD unable to } \\
\text { attend services in person? } \\
\text { 2. What barriers are there for PLWD accessing } \\
\text { dementia services? } \\
\text { 3. Can local or government incentives or policies } \\
\text { support vCST implementation? }\end{array}$ & $\begin{array}{l}\text { I. Some remote local and some national services for PLWD, such as telephone befriending and the Alzheimer's Society } \\
\text { helplines, but these were suspended during the COVID-19 lockdown or were limited in the support they offer. } \\
\text { 2. Barriers included: Awareness and navigation of services; having the means or technology to access them; worries about } \\
\text { interventions or feeling "assessed". Services should be advertised in an understandable, attractive and reassuring way. } \\
\text { 3. Not aware of policies to support vCST implementation. There are local policies to make areas dementia-friendly and } \\
\text { national policies to digitalise health care in the National Health Service (NHS) long-term plan. }\end{array}$ \\
\hline \multicolumn{3}{|r|}{ CFIR domain: “Inner Setting” } \\
\hline PLWD & $\begin{array}{l}\text { I. What factors would affect the likelihood that } \\
\text { PLWD would attend vCST? }\end{array}$ & $\begin{array}{l}\text { I. Increasing likelihood: Easily accessible sessions and at a convenient time of day. Participants are aware that others in the } \\
\text { group also have dementia. Other attendees live locally. Group facilitator has good interpersonal skills. Decreasing likelihood: } \\
\text { Poor internet connection or technology access/literacy. If PLWD is also a caregiver for someone else, they would not have the } \\
\text { time to attend. Concerns about meeting people for the first time online. }\end{array}$ \\
\hline Caregivers & $\begin{array}{l}\text { I. What factors would affect the likelihood that } \\
\text { PLWD would attend vCST? } \\
\text { 2. What are vCST's perceived benefits? }\end{array}$ & $\begin{array}{l}\text { I. Increasing likelihood: Knowing or remembering other group members on the screen. Watching recorded sessions as } \\
\text { a "taster". Sessions at a convenient time of day that capitalises alertness levels. Having regular session reminders. } \\
\text { 2. Decreasing likelihood: Larger group sizes and having a smaller screen. } \\
\text { 3. Social interaction, prompts conversation with partners/families, access for those in rural areas or unable to leave home. }\end{array}$ \\
\hline Facilitators & $\begin{array}{l}\text { I. What organisational resources are required to } \\
\text { run vCST? }\end{array}$ & $\begin{array}{l}\text { I. Time (for planning, adapting the sessions for online delivery, recruitment, group facilitation and administration) and having } \\
\text { access to the required technology, including a Zoom subscription. }\end{array}$ \\
\hline Service Managers & $\begin{array}{l}\text { I. How are decisions on dementia treatments } \\
\text { made centrally and locally? } \\
\text { 2. Is there any local support available for vCST } \\
\text { implementation? } \\
\text { 3. Is vCST implementation essential? }\end{array}$ & $\begin{array}{l}\text { I. Most NHS services offer evidence-based treatments/interventions from the NICE guidelines. Third sector providers have } \\
\text { flexibility to offer interventions based on service user need. Investments tend to be made for the acute stages of dementia (eg } \\
\text { bed space or care homes), rather than the early stages, which could be more cost-effective in the long term. } \\
\text { 2. There is no known local support for vCST implementation in services. Interventions such as vCST tended to be } \\
\text { implemented from the bottom up rather than through top-down investments. } \\
\text { 3. vCST implementation is essential in dementia services order to increase access for service users who are unable to travel. }\end{array}$ \\
\hline
\end{tabular}




\begin{tabular}{|c|c|c|}
\hline Stakeholders & Key Questions & Key Ideas \\
\hline \multicolumn{3}{|r|}{ CFIR domain "Individual" } \\
\hline PLWD & $\begin{array}{l}\text { I. How confident/able do PLWD feel using video } \\
\text { conferencing technology? } \\
\text { 2. What support is needed for PLWD to use video } \\
\text { conferencing? } \\
\text { 3. Are PLWD able to stay engaged? }\end{array}$ & $\begin{array}{l}\text { 1. Initially daunting for PLWD due to lack of experience using technology, but then perceived positively once it has been used. } \\
\text { It enables PLWD to see other people during pandemic restrictions and reduces feelings of isolation. } \\
\text { 2. Unable to access video conferencing technology independently. Support is needed from a friend, caregiver, or family } \\
\text { member, alongside the "how to guide". } \\
\text { 3. Able to engage in sessions vCST using video conferencing apps for } 60 \text { minutes without becoming tired or distracted. }\end{array}$ \\
\hline Caregivers & $\begin{array}{l}\text { 1. How confident/able do PLWD feel using video } \\
\text { conferencing technology? } \\
\text { 2. Barriers for PLWD using these apps? } \\
\text { 3. What support is needed for PLWD to use video } \\
\text { conferencing? } \\
\text { 4. Are PLWD able to stay engaged? What affects } \\
\text { their ability to engage? }\end{array}$ & $\begin{array}{l}\text { I. Video conferencing works well but requires caregivers to set up and support in most cases. Preference for Zoom over } \\
\text { other video conferencing apps due to prior experience. } \\
\text { 2. Concerns about privacy and security of the video conferencing apps, and sessions being interrupted by internet issues. } \\
\text { 3. Caregivers giving gentle encouragement to help PLWD to engage, and facilitators allowing flexibility for participants to "dip } \\
\text { in and out" of sessions if needed. } \\
\text { 4. PLWD may find it hard to concentrate or stay engaged for a I-hour session online. Factors affecting engagement include } \\
\text { severity of cognitive impairment, effective facilitation, caregiver supporting set up, and session attendance. }\end{array}$ \\
\hline Facilitators & $\begin{array}{l}\text { I. Are PLWD able to stay engaged in vCST using } \\
\text { video conferencing apps? }\end{array}$ & $\begin{array}{l}\text { I. PLWD can find it difficult to stay engaged for a I-hour session online (compared to in-person). Engagement can be } \\
\text { supported by breaking up the session, directing questions to participants who have not spoken, staying in "gallery mode" as } \\
\text { much as possible so group members can see each other talking rather than looking at images. }\end{array}$ \\
\hline $\begin{array}{l}\text { Facilitators and } \\
\text { Service Managers }\end{array}$ & $\begin{array}{l}\text { I. Do facilitators need training to run vCST } \\
\text { groups? }\end{array}$ & $\begin{array}{l}\text { I. Facilitators and Service Managers both noted that extra training would support group facilitators to run groups, especially } \\
\text { in using video conferencing apps/Zoom to run sessions, as facilitators may lack experience in using this technology. }\end{array}$ \\
\hline \multicolumn{3}{|r|}{ CFIR Domain: "Process" } \\
\hline PLWD & $\begin{array}{l}\text { I. What were the positives and negatives of } \\
\text { PLWD's experiences of } v C S T \text { ? }\end{array}$ & $\begin{array}{l}\text { I. Positives: Social component, enjoyable sessions with stimulating activities, more convenient to attend from home rather } \\
\text { than travel to services. Negatives: Difficulty viewing a small screen (large screen is needed), no physical contact with others } \\
\text { (preference for in-person CST, but vCST is better than nothing as it facilitates connection with others). }\end{array}$ \\
\hline Caregivers & $\begin{array}{l}\text { 1. How can caregivers support engagement?- } \\
\text { 2. Do caregivers feel they can leave the person } \\
\text { they care for to join in alone? }\end{array}$ & $\begin{array}{l}\text { I. Encouragement, giving reminders about attending the session, being present and prompting. Caregivers are essential for } \\
\text { reminding participants about sessions and to bring any objects } \\
\text { 2. Caregivers feel able to leave the person they care for to participate alone but would need to be nearby to offer technical } \\
\text { support if needed. }\end{array}$ \\
\hline
\end{tabular}


Table I (Continued).

\begin{tabular}{|c|c|c|}
\hline Stakeholders & Key Questions & Key Ideas \\
\hline \multicolumn{3}{|r|}{ CFIR Domain: “Process” (continued) } \\
\hline Facilitators & $\begin{array}{l}\text { I. What measures could to ensure that vCST } \\
\text { groups start on time? } \\
\text { 2. How many facilitators are required? } \\
\text { 3. How can distressed group members be } \\
\text { supported in vCST? } \\
\text { 4. What feedback has been received by PLWD } \\
\text { who have experienced vCST? }\end{array}$ & $\begin{array}{l}\text { I.Sending reminder emails on the day, inviting participants to log in } 10-15 \text { minutes early, practicing use of Zoom before } \\
\text { attending the group, and having a second facilitator to manage issues with technology. } \\
\text { 2.Two facilitators, one to lead the session and one to offer practical and emotional support to facilitators and participants. } \\
\text { 3. Distressed group members can be supported by the second facilitator in a separate breakout room whilst the lead } \\
\text { facilitator continues delivering the session and with a follow up telephone call after the session ends. } \\
\text { 4. PLWD would prefer in-person CST, but vCST is better than no intervention as it helps them feel less isolated. vCST should } \\
\text { continue to run past the pandemic to increase access for those living in rural areas or unable to leave the house }\end{array}$ \\
\hline Service Managers & $\begin{array}{l}\text { I. How can services support PLWD to engage in } \\
\text { vCST? } \\
\text { 2. Do services have the means to support vCST } \\
\text { implementation? }\end{array}$ & $\begin{array}{l}\text { I. Services can support PLWD engage by practising Zoom sessions beforehand to facilitate learning and confidence, and to } \\
\text { offer meetings to provide reassurance and answer questions about vCST before signing up. } \\
\text { 2. Services are generally able to provide the technology and resources to staff to be able to deliver the intervention. Two } \\
\text { facilitators are required in case there are technological or other resource issues. }\end{array}$ \\
\hline
\end{tabular}

Abbreviations: CST, Cognitive Stimulation Therapy; PLWD, Person/people living with dementia; vCST, Virtual Cognitive Stimulation Therapy. 
Table 2 vCST Session Themes

\begin{tabular}{|l|l|}
\hline I. Physical games & 8. Being creative \\
2. Sounds & 9. Categorising objects \\
3. Childhood & 10. Orientation \\
4. Food & 11. Using money \\
5. Current affairs & 12. Numbers games \\
6. Faces/Scenes & 13. Word games \\
7. Word association & 14. Team quiz \\
\hline
\end{tabular}

\section{Platform and Technology}

The video conferencing platform Zoom was chosen to deliver the sessions, as most people had experience with and preferred this platform. To support participants to access the sessions, a "How to use Zoom" guide was created, which should be sent to participants before the group. Participants should also be offered a one-to-one session on Zoom prior to attending the group to give them experience with using the platform, alongside additional telephone support as required. Participants should be advised to use a laptop or tablet and not a mobile phone to access the sessions, as the smaller screen of a mobile phone does not allow multiple participants to be viewed. Sessions should be set in "Gallery view" so that all participants are on screen simultaneously.

\section{Role of Facilitators}

Each group should run with two facilitators, one to lead on delivering the content and one to provide practical and other types of support as required. Reminder emails with the Zoom link should be sent to all participants the day before each session. Participants should be asked to sign into the session 10-15 minutes before the start time, to allow sessions to begin punctually and to allow time for the second facilitator to contact any participants who have not attended on time.

\section{Support from Caregiver}

If a participant is unable to access Zoom independently, the person living with dementia should identify a named caregiver with both of their consent who would support them to access the sessions and be contacted for any technical support. Any caregivers involved in giving support should be advised not to attend the sessions, but to be nearby (ie, in the next room) for the duration of the session in case they need to give technical support to the participant.

\section{Part 2: Field-Testing}

\section{Facilitators}

Field-testing took place across 10 groups in Brazil, Hong Kong, India, Ireland, and the UK. In Brazil a Postdoctoral Researcher, a Clinical Psychology student and a Gerontologist facilitated the sessions in a university setting. Three facilitators in Hong Kong and two in Ireland were Occupational Therapists in a university and care service setting respectively. In India, a Research Assistant and a Psychiatrist facilitated the sessions in a non-governmental organisation. In the UK, four facilitators were Trainee Clinical Psychologists, and one was a psychology PhD student, all based in a university. Facilitators were trained in CST, and were provided with guidelines for vCST delivery which had been developed from the focus group findings. ${ }^{29}$ All 15 facilitators were contacted, and feedback was received from 14.

\section{vCST Participants}

vCST participant demographics are not outlined in this paper, as this was a field-testing study. However all participants met the inclusion criteria for group CST, which comprises:

1. Meeting the ICD-10 criteria for dementia.

2. Having mild to moderate dementia (confirmed by the person with dementia and their caregiver or rated on the Clinical Dementia Rating Scale). ${ }^{30}$

3. Having sufficient hearing and vision to follow conversation and comment on visual material. 
4. Having the ability to participate in a group for 1 hour.

5. Additionally for vCST, participants needed access to technology, and the ability to use video conferencing software, or a caregiver who could support.

\section{Feedback from Field-Testing}

\section{Which Sessions/Activities Went Well?}

There was variety of feedback relating to the sessions and activities that worked best, due to group preferences or levels of engagement which could vary from session to session. However, there were some common themes across groups. Facilitators reported that sessions involving physical objects or "show and tell" had worked well, as the items sparked conversation and supported participant engagement. Some reiterated that participants needed to be reminded at the end of the previous session to bring physical objects for the following week, and suggested strategies such as encouraging participants to mark this in their diaries or sending a reminder email before the session. Structured activities or those with visual stimuli were reported to have run successfully. Examples of such activities include in "Faces and Scenes", participants compared photos of famous places in the past and present and discussed differences and similarities. In "Word Games" participants played "hangman" or completed the gaps of common sayings or idioms. In "Identifying Sounds" participants matched images of items and their associated sound clips presented through PowerPoint.

\section{Which Sessions/Activities Did Not Go As Well?}

Facilitators reported that, for some groups, discussion-based activities that were less structured were not as successful, especially in groups where participants had specific language or communication difficulties. For example, activities in the "Current Affairs" session, and the warm-up activity that involved discussing a recent news article. In some cases, this was attributed to group dynamics, where more reserved group members did not participate as much in discussions. Furthermore, some facilitators reported that it was challenging to find articles of interest, as the news was focused on the pandemic, which many participants and facilitators did not want to discuss every week.

Some activities had more complicated instructions, for example, the bingo activity in "Number Games" and the paper folding activity in "Being Creative", which also required fine motor dexterity and was more challenging for some participants. Some facilitators also fed back that it was more difficult to prompt or guide participants on activities where the task is completed outside the camera's field of view - for example searching for a number in "bingo" or colouring in - as the camera generally focuses on the face of the participant rather than on the table in front of the participant. Facilitators felt that these challenging activities worked better if a caregiver was available to support.

The group song that starts each session also had mixed feedback. Some facilitators fed back that groups disengaged with this activity, and one group wanted to play different songs each week instead of listening to the same song.

\section{Did Any Sessions Need Adapting Further?}

In India and Hong Kong, facilitators posted or couriered resource packs to participants, instead of relying on the "shared screen" function to show visual stimuli. These packs included images, worksheets and resources needed for the activities such as colour pencils and maps, and in India there was an accompanying instruction leaflet for caregivers sent over email. The printed resources could also include larger text and pictures for those who struggle to see the PowerPoint.

Some facilitators reported that overuse of the "shared screen" function reduced the quality of interaction, as participants' faces become small icons when function is enabled, which can be hard to see and make sense of. However, in other groups, the shared screen function worked effectively, and the visual stimuli enhanced engagement. Facilitators advised that "shared screen" is used for a limited period and that all resources for each session should be on one PowerPoint to avoid having to move from one screen to another.

\section{Did Any Specific Activities Need Adapting?}

Poor quality of internet connections was an issue for some groups, which could result in a lag between the images and sound when sharing a video. This was confusing for some participants, so some facilitators reverted to using a text or 
images as stimuli. Some facilitators adapted the "Current Affairs" section to include newspaper headlines, articles and images instead of videos. One facilitator reported that they had adapted the team quiz in Session 14 to an individual quiz, as they found it was harder for participants to confer in teams over Zoom.

\section{Did You Develop Any New Ideas?}

In Ireland, the facilitators fed back that participants had enjoyed the exercise videos from "Physical Games" so much, they incorporated physical activity into the warm-up of each session. Facilitators in Hong Kong made use of online resources and websites to provide multisensory stimulation, including a virtual tour of an art gallery for "Being Creative", or the use of street view in Google Maps to go for a "virtual walk" around a city or neighbourhood for "Orientation". Other activities were carried out that were similar in aims and delivery to the suggested activities, such as creating an "odd one out" game in the "Categorising Objects" session or listing as many words as possible from a category beginning with the same letter in the "Word Association" session. In India, groups took part in chair yoga during the "Physical Games" session and, based on the suggestion from a caregiver, participants were encouraged to wear traditional clothes in the last session to celebrate the cultural and ethnic diversity of the group, which promoted conversation amongst the group. In Brazil, the "Being Creative" session coincided with Mothering Sunday, so the activities were themed around motherhood. These new activities demonstrate the flexibility that CST facilitators can employ when planning sessions.

\section{Any Other Comments}

The majority of facilitators reiterated the importance of a trial session using videoconferencing technology for participants and caregivers, anda second facilitator to support participants join sessions and address any issues with technology. Caregivers were required to support with technology and, in some cases, with more complicated activities. In India, where participants only had access to tablet computers, it was recommended that groups comprised of three participants due to the smaller screen size and limited space for more faces.

During orientation at the start of each session, a facilitator in India reminded participants that the session was taking place virtually and gave a brief overview of the functions of Zoom. This helped to address disorientation related to using videoconferencing platforms and challenges posed by lower levels of digital literacy in older adults with dementia. The facilitators in Ireland noted that it was more challenging to use orientation sensitively using Zoom, as they had to rely on sharing images or using verbal cues, as opposed to facilitating in-person groups where orientation can be done subtly using props in the room, for example, using flowers such as daffodils to orientate to spring.

A pair of facilitators in the UK gave participants the option of staying on the Zoom call after meetings. They reported that the participants in one group requested this as a way of socialising, but it may not be suitable for all groups. In Ireland, the facilitators sent a follow up email with a summary of the session and included any materials and worksheets used. This also provided participants an opportunity to ask questions and share feedback between sessions.

After all feedback from facilitators was collated, the final version was reviewed and approved by all co-authors and a consensus was reached to develop the final vCST session protocol, which is outlined in Table 3 . The general guidelines for vCST delivery which had initially been developed from the focus group findings were updated based on further insights from field-testing, and are freely available online (https://www.ucl.ac.uk/international-cognitive-stimulationtherapy/cst-research/virtual-cst). ${ }^{29}$

\section{Discussion}

This study aimed to investigate the feasibility of a new vCST protocol for people living with dementia through stakeholder consultation and feedback from 14 facilitators following field-testing in the Brazil, China (Hong Kong), India, Ireland, and the UK. The qualitative findings indicate that the adapted protocol was feasible, acceptable to facilitators and had the flexibility to be adapted across cultures. The study therefore supported the proposal of a new delivery framework.

The proposed protocol and framework are vital, given that services have not been able to deliver face-to-face interventions during the COVID-19 pandemic. A recent survey found that during the height of the COVID-19 pandemic 
Table 3 Final vCST Session Protocol

\begin{tabular}{|c|c|}
\hline $\begin{array}{l}\text { Choices for } \\
\text { Main Activities }\end{array}$ & Resources Required \\
\hline \multicolumn{2}{|c|}{ Session I: Physical games } \\
\hline $\begin{array}{l}\text { I. Throw a virtual } \\
\text { ball } \\
\text { 2. Question and } \\
\text { answer - health } \\
\text { and exercise } \\
\text { 3. Chair exercise } \\
\text { 4. Sit-down dance } \\
\text { with the group } \\
\text { song. }\end{array}$ & $\begin{array}{l}\text { I. None required. } \\
\text { 2. Create a set of question cards in different colours/numbers (physical cards or on } \\
\text { PowerPoint). eg thoughts on healthy living; your favourite exercise; your exercise } \\
\text { routine. } \\
\text { 3. Use appropriate videos online or download and store on the computer in } \\
\text { advance. } \\
\text { 4. Find group song on internet or download to computer. }\end{array}$ \\
\hline
\end{tabular}

\section{Delivery Notes}

\section{Session 2: Sounds}

\begin{tabular}{l|l} 
I. Name the & I. Find sounds of different instruments online (eg, piano, violin, saxophone, etc).
\end{tabular} instrument.

2. Show and tell - $\quad$ 3. Find sounds online (eg, car, doorbell, dog, etc.), and add matching pictures to a musical 3. Find sounds online (eg,

instruments.

4. Find appropriate songs on internet.

I. Pretend to throw a ball and say something about yourself.

2. Participants take turns to choose one card from the facilitator and answer questions in turn. 3. Share screen and sound function required. Facilitate the exercise session yourself if the internet connectivity for video sharing is poor.

4. Facilitate group dancing. "Share sound" function required.

3. Match the pictures and sounds of everyday objects.

4. Listening to

songs.

\section{Session 3: Childhood}

\begin{tabular}{|l|l|l|l}
\hline I. Childhood & I. Create a worksheet with questions (eg birthplace, names of siblings, childhood
\end{tabular} memories $\quad$ address, favourite school subjects).

worksheet $\quad$ 2. Participants need pencil and paper or provide in pack.

2. Draw 3 3. Participant to bring available resources.

childhood homes 4. Create a PowerPoint or worksheet with images and show in share screen or 3. Show and tell - bring snacks and show on camera.

childhood

5. Create PowerPoint or print-out with pictures of childhood toys. Ask

memories

5. Create PowerPoint or print-out with pictures of
participants to bring childhood toys to the session.

I. Play the sounds of different instruments and ask participants to name them. "Share sound" required.

2. Ask members to bring musical instruments and show how to use it

3. Play the sounds to the group and show the PowerPoint on share screen. "Share sound" function required.

4. Play and discuss familiar songs. "Share sound" required.

4. Childhood

snacks

5. Childhood toys

I. Send worksheet in a resource pack, or for members to print. Give group time to answer questions, then discuss answers.

2. Participants draw their homes from memory and show group.

3. Group members to bring something that remind them of their childhood, eg, an object, a photo, or a food item etc.

4. Ask participants to describe the taste (is it sour or sweet?). Ask participants what their favourite snacks are nowadays.

5. Discuss what toys the groups" children or grandchildren play with. Ask how the toys nowadays differ from their own childhood toys. 
Delivery Notes

\begin{tabular}{|c|c|}
\hline $\begin{array}{l}\text { Choices for } \\
\text { Main Activities }\end{array}$ & Resources Required \\
\hline \multicolumn{2}{|l|}{ Session 4: Food } \\
\hline $\begin{array}{l}\text { I. Shopping task } \\
\text { 2. Ingredients } \\
\text { game } \\
\text { 3. Cookery } \\
\text { videos or recipes } \\
\text { 4. Show and tell - } \\
\text { food items }\end{array}$ & $\begin{array}{l}\text { I. Create a PowerPoint or worksheet with images and prices of food items. } \\
\text { 2. Create a PowerPoint or worksheet with images of food items. } \\
\text { 3. Find YouTube videos or recipes to show on share screen. } \\
\text { 4. Participant to bring available resources. }\end{array}$ \\
\hline
\end{tabular}

Session 5: Current affairs

\begin{tabular}{l|l}
\hline $\begin{array}{l}\text { I. Recent news } \\
\text { stories }\end{array}$ & $\begin{array}{l}\text { I. Find recent news videos, images, or headlines online. Ask participants to bring a } \\
\text { copy of a newspaper. }\end{array}$ \\
$\begin{array}{l}\text { 2. Discussion/ } \\
\text { voting on current } \\
\text { affairs }\end{array}$ & $\begin{array}{l}\text { 2. Prepare questions on topics eg, Thoughts on social media, retirement age, } \\
\text { telephone/ internet scams, sporting events. }\end{array}$ \\
$\begin{array}{l}\text { 3. Old and new } \\
\text { newspaper }\end{array}$ & $\begin{array}{l}\text { articles or headlines. } \\
\text { articles }\end{array}$
\end{tabular}

I. Ask members to shop for a theme from food items available (eg, breakfast/ lunch/ dinner preparation for a family of 4 ).

2. Ask participants to group foods and name the category and/or "make" a dish using different combination of ingredients.

3. View videos or recipes online and discuss. Ask participants to choose their favourite food or chef.

4. Ask members to bring some food items to sample that trigger particular memories. Discuss taste/texture/smell.

Session 6: Faces and scenes

\begin{tabular}{|c|c|c|}
\hline $\begin{array}{l}\text { I. Then and now } \\
\text { photos } \\
\text { 2. Photos of } \\
\text { people } \\
\text { 3. Virtual tour } \\
\text { 4. Movie clip }\end{array}$ & $\begin{array}{l}\text { I. Create a PowerPoint or worksheet with images. } \\
\text { 2. Create a PowerPoint or worksheet with images. } \\
\text { 3. Use Google Maps on internet browser and share screen. } \\
\text { 4. Video clip or movie song can be played on YouTube. }\end{array}$ & $\begin{array}{l}\text { I. Present photos that show the past and present of the same place. Participants compare and } \\
\text { describe the differences. } \\
\text { 2. Show one face at a time, ask open questions, eg "Do you like what their clothes?", "What } \\
\text { might they be doing/thinking?" Then show two or more faces together, ask questions eg, "Who } \\
\text { looks older/younger?", "What do they have in common?" } \\
\text { 3. If participants have finished the childhood home information in Session 3, use Google Maps } \\
\text { to show participants what places look like now. } \\
\text { 4. Watch a video clip or song from a famous movie and stimulate a discussion around the faces } \\
\text { in it and where it was set. }\end{array}$ \\
\hline
\end{tabular}

I. Discuss news story. "Share sound" required for video.

3. Facilitate discussion and encourage all to contribute. Compare the current situation with the situations the participants faced in the past 
Table 3 (Continued).

\begin{tabular}{|c|c|}
\hline $\begin{array}{l}\text { Choices for } \\
\text { Main Activities }\end{array}$ & Resources Required \\
\hline \multicolumn{2}{|c|}{ Session 7: Word association } \\
\hline $\begin{array}{l}\text { I. Fill in the } \\
\text { blanks } \\
\text { 2. Finish the song } \\
\text { lyrics } \\
\text { 3. Categories/ } \\
\text { themes of words }\end{array}$ & $\begin{array}{l}\text { I. Create PowerPoint or worksheet with phrases with blanks eg quantities (a cup } \\
\text { of...), or proverbs (A stitch in time...). } \\
\text { 2. Find appropriate songs online. } \\
\text { 3. None required. Can use shared whiteboard. }\end{array}$ \\
\hline
\end{tabular}

Delivery Notes

\section{Session 8: Being Creative}

I. Paper folding $\quad$ I. Participants bring paper/newspaper or provide in pack.

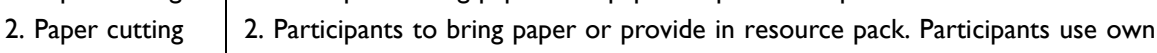

3. Drawing

scissors. Find video tutorials online.

portraits

3. Participants to bring paper/pencils or provide in pack.

Shared drawing 4. Participants to bring paper/pencils or provide in resource pack. Facilitator uses

5. Virtual tour of shared whiteboard.

$\begin{array}{ll}\text { art museum } & \text { 5. Find online video/images from local museum website. Create a PowerPoint or }\end{array}$ worksheet with images of artwork/paintings.

\section{Session 9: Categorising Objects}

I. Naming items

in a category

2. "Name-place-

animal-things"

game

3. Categorising

everyday objects

4. Show and tell -

household

objects

5. "Odd one out"

game

I. Show participants well-known sentences with blanks; ask the group to fill in the blanks. 2. Start songs and ask group to finish the lyrics. Use "Share sound".

3. Name a category/theme and ask group to say as many related-words as possible (eg animals, word starting with Z).

\section{Follow online videos (eg, paper airplane, simple origami)}

2. Lead the group to make some paper snowflakes and encourage them to develop their own designs.

3. Ask members to draw pictures of each other to share with the group.

4. Facilitator draws on shared whiteboard to. Group follow along and draw on a paper, then share with group.

5. Discuss artwork as a group. Suitable for a group who might struggle with drawing.

Use virtual whiteboard

2. Participants require pen and paper.

3. Create PowerPoint/worksheet with pictures of 10 everyday objects.

4. Participant to bring available resources

5. Create a PowerPoint or worksheet with images.
I. Ask the group to come up with a category/choose from a list (eg animals, foods) and name items in the category.

2. Pick a letter at random and ask participants to write down a name, place, animal, and a thing starting with that letter. Discuss responses.

3. Ask participants to group the objects based on different categories (eg, colour, usage, or where to find them).

4. Ask the group to find objects from home that are a certain colour, then categorise the items into groups.

5. Show 3 or 4 pictures (eg animal, food, faces) at the same time, and ask the group to point out the odd one out and why. 


\section{Delivery Notes}

Main Activities

Resources Required

\section{Session 10: Orientation}

\begin{tabular}{l|l} 
I. Country map & I. Provide a country map to print or send in resource pack.
\end{tabular}

2. Travel

2. Provide a country map to print or send in resource pack.

recommendations 3. Provide a world map to print or send in a resource pack.

I. Participants find/mark where they live or were born. Discuss whether they moved from an area to another, and how areas have changed.

2. Suggest places to someone visiting country for the first time.

3. World map

3. Find/mark countries members have visited or would like to visit

\section{Session II: Using Money}

\begin{tabular}{l|l|} 
I. Price guessing & I. Create PowerPoint or worksheet with images of items (eg things to buy for a
\end{tabular} task summer holiday).

2. Price matching 2. Create PowerPoint or worksheet with images of items and a list of their prices. task

3. None required.

3. Price of items 4. Make PowerPoint or worksheet with images of old and new banknotes, and

discussion newer payment methods (eg credit cards).

4. Comparing old 5. Make PowerPoint or worksheet with images of items and prices.

and new money

5. Online group

shopping game

\section{Session I2: Numbers games}

I. Adding up card $\quad$ I. Facilitator and participants need to have a deck of playing cards each.

values game

2. Bingo

3. Higher or 3. Facilitator to bring a deck of playing cards.

2. Use online bingo number generator and find bingo cards online (send to members to print out or send in pack).

lower card game 4. Participants need to have a deck of playing cards.

I. Ask participants to guess the price of each object and total price. Ask the group which object is most expensive / least expensive.

2. Ask participants to match the price of each object with the prices

3. Discuss how price of everyday items has changed in past 30 years.

4. Discuss how the money has changed, and how the value of money has changed.

5. Ask participants to make a shopping list with a budget, based on a context (eg, picnic,

preparing a hot-pot meal, hosting a party)

4. Snap card game

I. Randomly draw 2 playing cards for each participant or ask them to draw 2 from their own pack of cards. Ask them to add up the points of their cards. The member who gets the largest point wins.

2. Read out numbers from the online bingo number generator. Participants should cross out numbers on their bingo cards.

3. Participants take turns to guess if next card will be higher or lower.

4. Members take a card off the top of their pile and hold it to the camera. Anyone with matching cards says snap and gets a point. 
Table 3 (Continued).

\begin{tabular}{|c|c|c|}
\hline $\begin{array}{l}\text { Choices for } \\
\text { Main Activities }\end{array}$ & Resources Required & Delivery Notes \\
\hline \multicolumn{3}{|c|}{ Session 13: Word games } \\
\hline $\begin{array}{l}\text { I. Describe the } \\
\text { word } \\
\text { 2. Riddles } \\
\text { 3. Rebus game } \\
\text { 4. Hangman or } \\
\text { crossword game }\end{array}$ & $\begin{array}{l}\text { I. Create a list of words for participants to choose from. } \\
\text { 2. Prepare riddles or ask the group to share riddles they know. } \\
\text { 3. Find visual riddles/rebus puzzles online. Add to slides/worksheet. } \\
\text { 4. Find crossword or hangman online games. }\end{array}$ & $\begin{array}{l}\text { I. One participant chooses a word and describes it to others without saying word itself. } \\
\text { Members take turn as listeners and speakers. } \\
\text { 2. Solve the riddles as a group. } \\
\text { 3. Ask the group to guess the idioms depicted in the pictures. } \\
\text { 4. Share screen and ask al group members to participate. }\end{array}$ \\
\hline \multicolumn{3}{|c|}{ Session 14: Team quiz } \\
\hline $\begin{array}{l}\text { I.Group } \\
\text { competition (eg } \\
\text { quiz or favourite } \\
\text { game from past } \\
\text { sessions) } \\
\text { 2. Recap of } \\
\text { sessions }\end{array}$ & $\begin{array}{l}\text { I. Create a true or false or "myth buster quiz" (or find one online) or reuse } \\
\text { resources from previous sessions. } \\
\text { 2. None required. } \\
\text { For both activities: Participants bring tea/snacks to enjoy together as a celebration. }\end{array}$ & $\begin{array}{l}\text { I. Put group members into teams, or if it is difficult for them to confer it can be an individual } \\
\text { quiz. } \\
\text { 2. Discuss how participants have found the group i.e. which sessions did they like best/least. }\end{array}$ \\
\hline
\end{tabular}


in the UK, many National Health Service (NHS) memory clinics and third-sector dementia organisations did not have guidance on how to adapt CST for virtual delivery. This resulted in individual adaptation of CST by different facilitators, which was time-consuming and could result in inconsistencies across services (Fisher et al., In preparation).

Despite CST being a popular, widely available, and beneficial psychosocial intervention for people living with dementia, it still remains inaccessible to those who may be unable to travel due to health, socioeconomic or geographical reasons, as well as to those shielding during the COVID-19 pandemic. Access to virtual services is key to filling this gap. However, low levels of digital literacy and access to technology may be a barrier for older adults with dementia to access vCST. Access to virtual care is limited in low- and middle-income countries, and in the UK, there are 5.3 million "internet non-users", with those over the age of 75 years old making up over half of these non-users. ${ }^{31,32}$ Despite the shift to digital delivery of services, there is there is little evidence that those who were digitally excluded before the pandemic have become internet users since the pandemic. ${ }^{33}$ There is a clear need for top-down investment and engagement with older people to prevent digital exclusion. To support access to vCST on an individual level support from a caregiver and facilitator is necessary, including resolving technical issues and conducting a trial run using the video conferencing software. These approaches were also highlighted in a recent review of barriers and facilitators to telemedicine in dementia care. ${ }^{23}$

In vCST, unlike in face-to-face CST, participants of the same group can reside in different regions of a country. This not only makes CST accessible to a wider range of participants but may be a point of conversation and more stimulating cognitively for participants. However the facilitator should ensure that no participants are excluded and that different cultures are celebrated.

Previous research has found that memory, comprehension of syntax, and orientation are the cognitive domains the most impacted by face-to-face CST. ${ }^{34}$ However vCST may not have a comparable impact on these domains, due to the different method of delivery. Participants attend virtual sessions from home, remaining close to their caregivers and separate from the other participants and facilitators. This may impact the quality of the interaction with others and could affect the way that participants perceive themselves and fellow participants.

\section{Strengths and Limitations}

There are several strengths identified in the study, including the use of stakeholder consultation in the development of the final vCST guidelines and protocol. This is especially important given that both the people living with dementia and the group facilitators had both experienced vCST sessions prior to attending the focus groups. Stakeholder consultation is essential in creating new interventions as it can help to identify problems, solutions and priorities in the development and implementation process that researchers may not be aware of. ${ }^{35}$ Secondly, the protocol was field-tested in a variety of settings across five countries. CST is an internationally implemented intervention, and it is promising that virtual CST was acceptable and feasible across the five countries. Finally, the vCST protocol is adapted from the original CST inperson protocol developed by Spector et al., ${ }^{26}$ which has a strong evidence base for benefitting people living with dementia. ${ }^{2,36}$ As the vCST protocol adheres so closely to the original it makes comparisons across modality easily viable.

Despite these clear strengths, some limitations were also identified. The field-testing highlights that the protocol is feasible and acceptable, however the field-testing took place with only a selected group and many people will not have access or be able to engage in vCST groups due to lack of access to technology, income, or limited digital literacy. Sampling bias may have prevented reaching people with additional barriers for access to vCST, and future studies should try to recruit more inclusive samples. We did not use thematic analysis or other formal methods to analyse qualitative data, because there was a need to collect and analyse data rapidly at a time of limited staff capacity. Additionally, stakeholder consultation for the development of vCST was restricted to Hong Kong, India, and the UK. Due to limited time and staff capacity, this was compounded by having only one focus group per stakeholder group. Further focus groups could take place, adding more sites and using more detailed surveys, to elicit a wider range of views. Finally, the current study represents pilot work, with empirical testing of the intervention needed, including outcome measures and qualitative interviews. 


\section{Future Research}

Reporting of results from ongoing RCTs is required to establish the efficacy of vCST and to establish feasibility and acceptability, and the impact on different dementia populations. Trials of face-to-face CST groups have demonstrated benefits on mood and quality of life, and it is necessary to examine if these benefits remain when the intervention is delivered virtually. Future research could also measure the facilitators' fidelity to the vCST protocol. Fidelity to an intervention increases the reliability and validity of the data as all participants are more likely to receive the same intervention. Data on participant engagement in vCST sessions could also be explored, which would help to inform if the vCST protocol needs to be adjusted further, for example if people living with dementia are not able to engage in 45 to 60-minute session. Participant self-report measures may not be an accurate indicator of engagement, so observational data could be collected through the development of a coding system for researchers to use when watching video recordings of sessions, or by using eye-tracking technology to assess eye movements which can correlate with participant attention. ${ }^{37}$

\section{Conclusion}

Overall, a 14-session vCST protocol developed in this study was feasible and acceptable as a psychosocial, e-health intervention for people living with dementia. We therefore recommend that vCST is offered as an intervention across dementia services to increase access to a CST programme for those who are otherwise unable to access CST in-person, for reasons including health, mobility, and transport problems. This is especially important when services are not able to offer in-person CST due to social distancing needs during current and future pandemics. vCST may not replace in-person CST sessions but can be a useful alternative in situations where in-person CST is not possible. Larger trials on vCST are required to further investigate benefits on mood and quality of life.

\section{Abbreviations}

CBT, Cognitive Behavioural Therapy; CFIR, Consolidated Framework for Implementation Research; CST, Cognitive Stimulation Therapy; NHS, National Health Service; NICE, National Institute for Health and Social Care Excellence; PLWD, Person/people living with dementia; vCST, Virtual Cognitive Stimulation Therapy.

\section{Acknowledgments}

We would like to thank the focus group participants, vCST group participants, and all vCST facilitators in Brazil, Hong Kong, India, Ireland, and the UK who field-tested and provided feedback on the protocol.

\section{Funding}

The work carried out in Brazil and India and supported by is supported by the following Global Alliance for Chronic Diseases (GACD) funding agencies: The United Kingdom Medical Research Council (MRC: MR/S004009/1) and the Indian Council of Medical Research (ICMR: Indo-foreign/67/M/2018-NCD-I). No funding bodies were involved in the design, collection, analysis, interpretation or writing of the research or manuscript.

\section{Disclosure}

AS offers Cognitive Stimulation Therapy (CST) training courses on a consultancy basis. The authors report no other conflicts of interest in this work.

\section{References}

1. Mauricio R, Benn C, Davis J, et al. Tackling gaps in developing life-changing treatments for dementia. Alzheimers Dement. 2019;5:241-253. doi:10.1016/j.trci.2019.05.001

2. Aguirre E, Woods RT, Spector A, Orrell M. Cognitive stimulation for dementia: a systematic review of the evidence of effectiveness from randomised controlled trials. Ageing Res Rev. 2013;12(1):253-262. doi:10.1016/j.arr.2012.07.001

3. Spector A, Thorgrimsen L, Woods B, et al. Efficacy of an evidence-based cognitive stimulation therapy programme for people with dementia: randomised controlled trial. Br J Psychiatr. 2003;183(3):248-254. doi:10.1192/bjp.183.3.248 
4. National Institute for Health and Care Excellence. Overview | dementia: assessment, management and support for people living with dementia and their carers | guidance | NICE; 2018. Available from: https://www.nice.org.uk/guidance/ng97. Accessed October 5, 2021.

5. Bertrand E, Naylor R, Laks J, Marinho V, Spector A, Mograbi DC. Cognitive stimulation therapy for Brazilian people with dementia: examination of implementation' issues and cultural adaptation. Aging Ment Health. 2019;23(10):1400-1404. doi:10.1080/13607863.2018.1488944

6. Wong GHY, Yek OPL, Zhang AY, Lum TYS, Spector A. Cultural adaptation of cognitive stimulation therapy (CST) for Chinese people with dementia: multicentre pilot study. Int J Geriatr Psychiatry. 2018;33(6):841-848. doi:10.1002/gps.4663

7. Alzheimer's Disease International. World Alzheimer report 2011: the benefits of early diagnosis and intervention; 2011. Available from: https:// www.alzint.org/resource/world-alzheimer-report-2011/. Accessed October 5, 2021.

8. Giebel C, Cannon J, Hanna K, et al. Impact of COVID-19 related social support service closures on people with dementia and unpaid carers: a qualitative study. Aging Ment Health. 2021;25(7):1281-1288. doi:10.1080/13607863.2020.1822292

9. Cuffaro L, Di Lorenzo F, Bonavita S, Tedeschi G, Leocani L, Lavorgna L. Dementia care and COVID-19 pandemic: a necessary digital revolution. Neurol Sci. 2020;41(8):1977-1979. doi:10.1007/s10072-020-04512-4

10. Dores AR, Geraldo A, Carvalho IP, Barbosa F. The use of new digital information and communication technologies in psychological counseling during the COVID-19 pandemic. IJERPH. 2020;17(20):7663. doi:10.3390/ijerph17207663

11. World Health Organization. Fifty-eighth world health assembly resolutions and decisions annex; 2005. Available from: https://apps.who.int/gb/ ebwha/pdf_files/WHA58-REC1/english/A58_2005_REC1-en.pdf. Accessed October 5, 2021.

12. Fairburn CG, Patel V. The impact of digital technology on psychological treatments and their dissemination. Behav Res Ther. 2017;88:19-25. doi:10.1016/j.brat.2016.08.012

13. Donker T, Bennett K, Bennett A, et al. Internet-delivered interpersonal psychotherapy versus internet-delivered cognitive behavioral therapy for adults with depressive symptoms: randomized controlled noninferiority trial. $J$ Med Internet Res. 2013;15(5):e82. doi:10.2196/jmir.2307

14. Pots WTM, Fledderus M, Meulenbeek PAM, Ten Klooster PM, Schreurs KMG, Bohlmeijer ET. Acceptance and commitment therapy as a web-based intervention for depressive symptoms: randomised controlled trial. Br J Psychiatr. 2016;208(1):69-77. doi:10.1192/bjp.bp.114.146068

15. Burton RL, O'Connell ME. Telehealth rehabilitation for cognitive impairment: randomized controlled feasibility trial. JMIR Res Protoc. 2018;7(2): e43. doi:10.2196/resprot.9420

16. LaMonica HM, English A, Hickie IB, et al. Examining internet and eHealth practices and preferences: survey study of Australian older adults with subjective memory complaints, mild cognitive impairment, or dementia. J Med Internet Res. 2017;19(10):e7981. doi:10.2196/jmir.7981

17. Lazar A, Thompson H, Demiris G. A systematic review of the use of technology for reminiscence therapy. Health Educ Behav. 2014;41 (1_suppl):51S-61S. doi:10.1177/1090198114537067

18. Pywell J, Vijaykumar S, Dodd A, Coventry L. Barriers to older adults' uptake of mobile-based mental health interventions. Digi Health. 2020;6:2055207620905422. doi:10.1177/2055207620905422

19. Wuthrich VM, Frei J. Barriers to treatment for older adults seeking psychological therapy. Int Psychogeriatr. 2015;27(7):1227-1236. doi:10.1017/ S1041610215000241

20. Charness N, Boot WR. Aging and information technology use: potential and barriers. Curr Dir Psychol Sci. 2009;18(5):253-258. doi:10.1111/ j.1467-8721.2009.01647.x

21. Peel N, Russell T, Gray L. Feasibility of using an in-home video conferencing system in geriatric rehabilitation. $J$ Rehabil Med. 2011;43 (4):364-366. doi:10.2340/16501977-0675

22. Di Carlo F, Sociali A, Picutti E, et al. Telepsychiatry and other cutting-edge technologies in COVID-19 pandemic: bridging the distance in mental health assistance. Int J Clin Pract. 2021;75(1). doi:10.1111/ijcp.13716

23. Yi JS, Pittman CA, Price CL, Nieman CL, Oh ES. Telemedicine and dementia care: a systematic review of barriers and facilitators. $J$ Am Med Dir Assoc. 2021;22(7):1396-1402.e18. doi:10.1016/j.jamda.2021.03.015

24. Cheung G, Peri K. Challenges to dementia care during COVID-19: innovations in remote delivery of group cognitive stimulation therapy. Aging Ment Health. 2021;25(6):977-979. doi:10.1080/13607863.2020.1789945

25. Craig P, Dieppe P, Macintyre S, Michie S, Nazareth I, Patticrew M. Developing and Evaluating Complex Interventions. BMJ. $2019: 21: 337$.

26. Spector A, Thorgrimsen L, Woods RT, Orrell M. Making a difference: an evidence-based group programme to offer Cognitive Stimulation Therapy (CST) to people with dementia. Hawker Publications; 2020. Available from: https://research.bangor.ac.uk/portal/en/researchoutputs/makingA-difference-an-evidencebased-group-programme-to-offer-cognitive-stimulation-therapy-cst-to-people-with-dementia(95a76b66-4d14-4580-894f97c920faeb68).html. Accessed October 5, 2021.

27. Damschroder LJ, Aron DC, Keith RE, Kirsh SR, Alexander JA, Lowery JC. Fostering implementation of health services research findings into practice: a consolidated framework for advancing implementation science. Implementation Sci. 2009;4(1):50. doi:10.1186/1748-5908-4-50

28. Raghuraman S, Lakshminarayanan M, Vaitheswaran S, Rangaswamy T. Cognitive stimulation therapy for dementia: pilot studies of acceptability and feasibility of cultural adaptation for India. Am J Geriatr Psychiatr. 2017;25(9):1029-1032. doi:10.1016/j.jagp.2017.04.014

29. International Cognitive Stimulation Therapy (CST) Centre website. International Cognitive Stimulation Therapy (CST) Centre: CST research virtual CST. International Cognitive Stimulation Therapy (CST) Centre; 2021. Available from: https://www.ucl.ac.uk/international-cognitivestimulation-therapy/cst-research/virtual-cst. Accessed November 9, 2021.

30. Morris JC. Clinical dementia rating: a reliable and valid diagnostic and staging measure for dementia of the Alzheimer type. Int Psychogeriatr. 1997;9(S1):173-176. doi:10.1017/S1041610297004870

31. Comas-Herrera A, Lorenz-Dant K, Ferri C, et al. Supporting People Living with Dementia and Their Carers in Low-and Middle-Income Countries During COVID-19. LTCcovid. Org. 2020:4.

32. Office for National Statistics. Exploring the UK's digital divide - Office for National Statistics; 2019. Available from: https://www.ons.gov.uk/ peoplepopulationandcommunity/householdcharacteristics/homeinternetandsocialmediausage/articles/exploringtheuksdigitaldivide/2019-03-04. Accessed October 5, 2021.

33. Age UK. Digital inclusion and older people - how have things changed in a Covid-19 world?; 2021. Available from: https://www.ageuk.org.uk/ globalassets/age-uk/documents/reports-and-publications/reports-and-briefings/active-communities/digital-inclusion-in-the-pandemic-final-march -2021.pdf. Accessed October 5, 2021.

34. Hall L, Orrell M, Stott J, Spector A. Cognitive stimulation therapy (CST): neuropsychological mechanisms of change. Int Psychogeriatr. 2013;25 (3):479-489. doi:10.1017/S1041610212001822 
35. O'Cathain A, Croot L, Duncan E, et al. Guidance on how to develop complex interventions to improve health and healthcare. BMJ Open. 2019;9 (8):e029954. doi:10.1136/bmjopen-2019-029954

36. Lobbia A, Carbone E, Faggian S, et al. The efficacy of Cognitive Stimulation Therapy (CST) for people with mild-to-moderate dementia. Eur Psychol. 2019;24(3):257-277. doi:10.1027/1016-9040/a000342

37. Parekh V, Foong PS, Zhao S, Subramanian R. AVEID: automatic video system for measuring engagement in dementia. In: 23 rd International Conference on Intelligent User Interfaces; ACM; 2018:409-413. doi:10.1145/3172944.3173010

\section{Publish your work in this journal}

Clinical Interventions in Aging is an international, peer-reviewed journal focusing on evidence-based reports on the value or lack thereof of treatments intended to prevent or delay the onset of maladaptive correlates of aging in human beings. This journal is indexed on PubMed Central, MedLine, CAS, Scopus and the Elsevier Bibliographic databases. The manuscript management system is completely online and includes a very quick and fair peer-review system, which is all easy to use. Visit http://www.dovepress.com/testimonials.php to read real quotes from published authors.

Submit your manuscript here: https://www.dovepress.com/clinical-interventions-in-aging-journal 